\title{
IMPROVEMENTS IN SINGLE PARTICLE CRYO-EM: 11Å STRUCTURE OF 290 kDa XDH REFINED FROM MULTIPLE 3D RECONSTRUCTIONS.
}

\author{
D.R. Beniac, T.Iwasaki ${ }^{* *}$, B.T. Eger ${ }^{*}$, E.F. Pai, F.P. Ottensmeyer
}

Ontario Cancer Institute and Department of Medical Biophysics, University of Toronto, 610 University Avenue, Toronto, ON, Canada, M5G 2N9; * Department of Biochemistry and Molecular Biology, Nippon Medical School, Sendagi, 1-1-5, Japan; ** Department of Biochemistry, University of Toronto, 1 King's College Circle, Toronto, ON, Canada, M5s 1 A8.

X-ray crystallography or nuclear magnetic resonance conventionally have solved threedimensional (3D) macromolecular structures. Recent advances in cryo-EM image analysis of single particles have made this an alternative method to solve 3D structures. In this study a method is presented which uses multiple 3D-EM reconstructions to produce a final average structure for the molecule being investigated.

A Scanning Transmission Electron Microscope (STEM) VG HB601UX was used in this investigation to image sets of individual freeze-dried molecules of xanthine dehydrogenase (XDH) at low temperature $\left(140^{\circ} \mathrm{C}\right)$ and under low dose conditions $\left(10.8 \mathrm{e} / \AA^{2}\right)$. The STEM has been configured with multiple detectors, and a Gatan PEELS spectrometer, which permit simultaneous acquisition of both elastic and inelastic scattered electrons. These paired dark field images are then combined computationally to produce images with a high signal to noise ratio. The molecular mass of XDH was calculated to be $289 \pm 94 \mathrm{kDa}$ for the population (Fig. A). From the initial population of 2313 single particle images 1621 were selected based on molecular mass. All subsequent 3D reconstructions were calculated using these selected images, which have a mass close to the $290 \mathrm{kDa}$ mass for the intact XDH dimer. The 1621 single particle images were then subdivided into independent sets used to produce 16 unique reconstructions via the Iterative Quaternion-Assisted Angle Determination (IQAD)(1) procedure. IQAD produces reference-free 3D reconstructions directly from unaveraged single molecule images. The 16 reconstructions were then aligned to each other, and multivariate statistical analysis and hierarchical ascendant classification were used to analyze the 16 structures. The dendrogram produced by the classification (Fig. B) was used to define distinct classes (1-6). The two reconstructions in class 5 are shown in Fig. C. The reconstructions in each class were then merged using a modification of the projection matching procedure (2). This method uses aligned projections from the multiple reconstructions in each class to produce a final weighted average structure. The weighted average reconstructions are presented in Fig. D for classes 1,2,4, and 5. These four structures were then subjected to a second round of projection-match-based merging to produce the final reconstruction shown in Fig. E. The structure is presented with three increasing density thresholds to highlight the interior mass density in the final average structure. The resolution of this final structure was estimated to be $11 \AA$ by phase residual analysis between separate final reconstructions from independent images. Fig. $\mathrm{F}$ is a ribbon diagram of XDH (1FO4.PDB) (3) that was determined by x-ray crystallography. This structure was not used in the analysis, but does serve as a reference for comparison to the structures that we have determined.

\section{References}

1. N.A. Farrow, F.P. Ottensmeyer, J. Opt. Soc. Am. A9, 1749 (1992)

2. Penczek et al., Ultramicroscopy 53, 251 (1994)

3. C. Enroth, et al., PNAS. 97, 10723 (2000) 

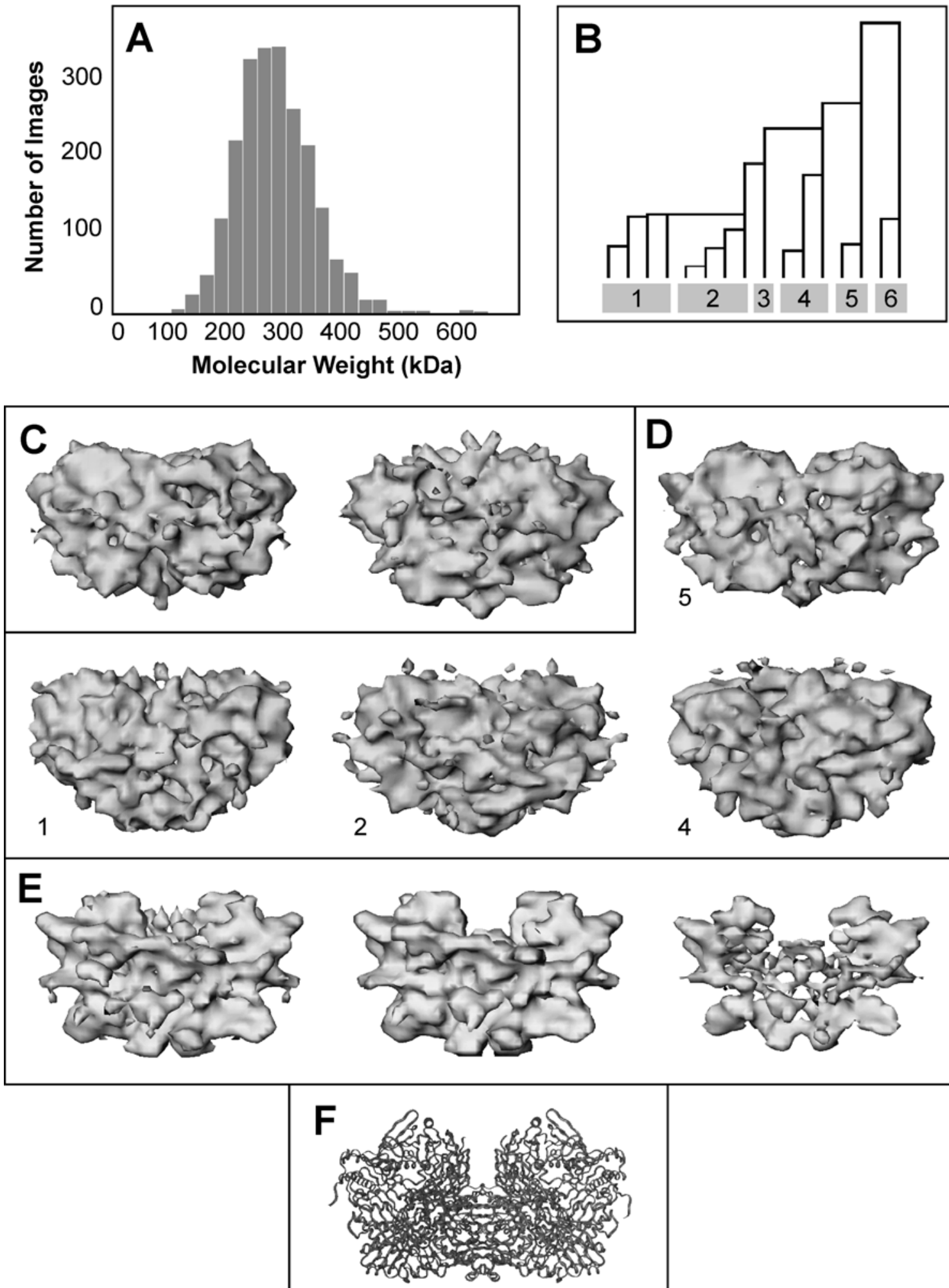